Marquette University

e-Publications@Marquette

2009

\title{
Deflection of a Viscoelastic Cantilever under a Uniform Surface Stress: Applications to Static-mode Microcantilever Sensors Undergoing Adsorption
}

\author{
Michael J. Wenzel \\ Marquette University \\ Fabien Josse \\ Marquette University, fabien.josse@marquette.edu \\ Stephen M. Heinrich \\ Marquette University, stephen.heinrich@marquette.edu
}

Follow this and additional works at: https://epublications.marquette.edu/civengin_fac

Part of the Civil and Environmental Engineering Commons

\section{Recommended Citation}

Wenzel, Michael J.; Josse, Fabien; and Heinrich, Stephen M., "Deflection of a Viscoelastic Cantilever under a Uniform Surface Stress: Applications to Static-mode Microcantilever Sensors Undergoing Adsorption" (2009). Civil and Environmental Engineering Faculty Research and Publications. 72.

https://epublications.marquette.edu/civengin_fac/72 


\title{
Deflection of a viscoelastic cantilever under a uniform surface stress: Applications to static-mode microcantilever sensors undergoing adsorption
}

\author{
M. J. Wenzel, ${ }^{1}$ F. Josse, ${ }^{1, a)}$ and S. M. Heinrich ${ }^{2}$ \\ ${ }^{1}$ Department of Electrical and Computer Engineering, Marquette University, P.O. Box 1881, Milwaukee, \\ Wisconsin 53201-1881, USA \\ ${ }^{2}$ Department of Civil and Environmental Engineering, Marquette University, P.O. Box 1881, Milwaukee, \\ Wisconsin 53201-1881, USA
}

(Received 19 September 2008; accepted 16 January 2009; published online 18 March 2009)

\begin{abstract}
The equation governing the curvature of a viscoelastic microcantilever beam loaded with a uniform surface stress is derived. The present model is applicable to static-mode microcantilever sensors made with a rigid polymer, such as SU-8. An analytical solution to the differential equation governing the curvature is given for a specific surface stress representing adsorption of analyte onto the viscoelastic beam's surface. The solution for the bending of the microcantilever shows that, in many cases, the use of Stoney's equation to analyze stress-induced deflection of viscoelastic microcantilevers (in the present case due to surface analyte adsorption) can lead to poor predictions of the beam's response. It is shown that using a viscoelastic substrate can greatly increase sensitivity (due to a lower modulus), but at the cost of a longer response time due to viscoelastic creep in the microcantilever. In addition, the effects of a coating on the cantilever are considered. By defining effective moduli for the coated-beam case, the analytical solution for the uncoated case can still be used. It is found that, unlike the case of a silicon microcantilever, the stress in the coating due to bending of a polymer cantilever can be significant, especially for metal coatings. The theoretical results presented here can also be used to extract time-domain viscoelastic properties of the polymer material from beam response data. (C) 2009 American Institute of Physics.
\end{abstract}

[DOI: $10.1063 / 1.3086626]$

\section{INTRODUCTION}

Currently, microcantilevers are being extensively investigated as potential chemical and biological sensor platforms to meet the need for real-time environmental monitoring as well as to perform medical diagnostic tests. The microcantilever's projected high sensitivity and the ability to design a very small array employing a large number of sensors have been the main driving factors behind this research. ${ }^{1}$ Detection limits at the parts-per-trillion level as well as single molecule detection have been demonstrated. ${ }^{2-5}$

Microcantilever sensors may be operated in either the dynamic (resonant) or the static mode. In the dynamic mode, mass loading and viscoelastic changes in the coating cause a measurable shift in the resonant frequency and quality factor. ${ }^{6-8}$ When operated in the static mode, expansion of the coating caused by absorbed anlayte or a surface stress caused by analyte adsorption onto the coating causes the microcantilever to bend. ${ }^{7,9,10}$ The sorption process (either absorption or adsorption) determines how the deflection is analyzed. Deflection caused by absorption-induced coating expansion is similar to the bending of a bimetal thermostat. As a result, the bimetal thermostat analysis by Timoshenko ${ }^{11}$ is often used to predict the deflection of the microcantilever caused by analyte absorption. Recently, it has been shown that when using a polymer coated microcantilever sensor it is often necessary to account for the viscoelastic properties of the

\footnotetext{
a) Author to whom correspondence should be addressed. Electronic mail: fabien.josse@marquette.edu.
}

coating. This leads to a more complex behavior and the deflection may exhibit an overshoot during absorption. ${ }^{12}$ Deflection caused by adsorption-induced surface stress is generally analyzed using Stoney's equation, ${ }^{13,14}$

$$
w_{\text {tip }}=\frac{3 L^{2}(1-\nu) \sigma_{s}}{E_{1} h_{1}^{2}},
$$

where $w_{\text {tip }}$ is the deflection at the tip of the cantilever, $L$ is the length of the cantilever, $\sigma_{s}$ is the surface stress, and $E_{1}$, $h_{1}$, and $\nu$ are the Young's modulus, thickness, and Poisson's ratio of the base layer, respectively. In the present paper, it will be convenient to represent the cantilever material by its biaxial modulus, $M_{1}=E_{1} /(1-\nu)$, rather than the Young's modulus, $E_{1}$.

In the static mode, the need for higher sensitivity to surface stress resulted in an increased interest in polymer-based microcantilevers, where the cantilever itself is made of a relatively rigid polymer with good chemical resistance such as SU-8. ${ }^{15,16}$ These glassy polymers offer less resistance to adsorption-induced surface stress compared to silicon, thus enhancing the sensitivity. However, using a polymeric cantilever has the added complexity of a time-dependent relaxation modulus, which is generally not considered when analyzing the deflection with Stoney's equation. To obtain a generalized form of Stoney's equation, which takes into account the viscoelasticity of the beam, the polymer material is represented as a three-parameter solid. The model describes the bending of a viscoelastic beam loaded with a surface stress. In some cases, the viscoelastic behavior of the canti- 

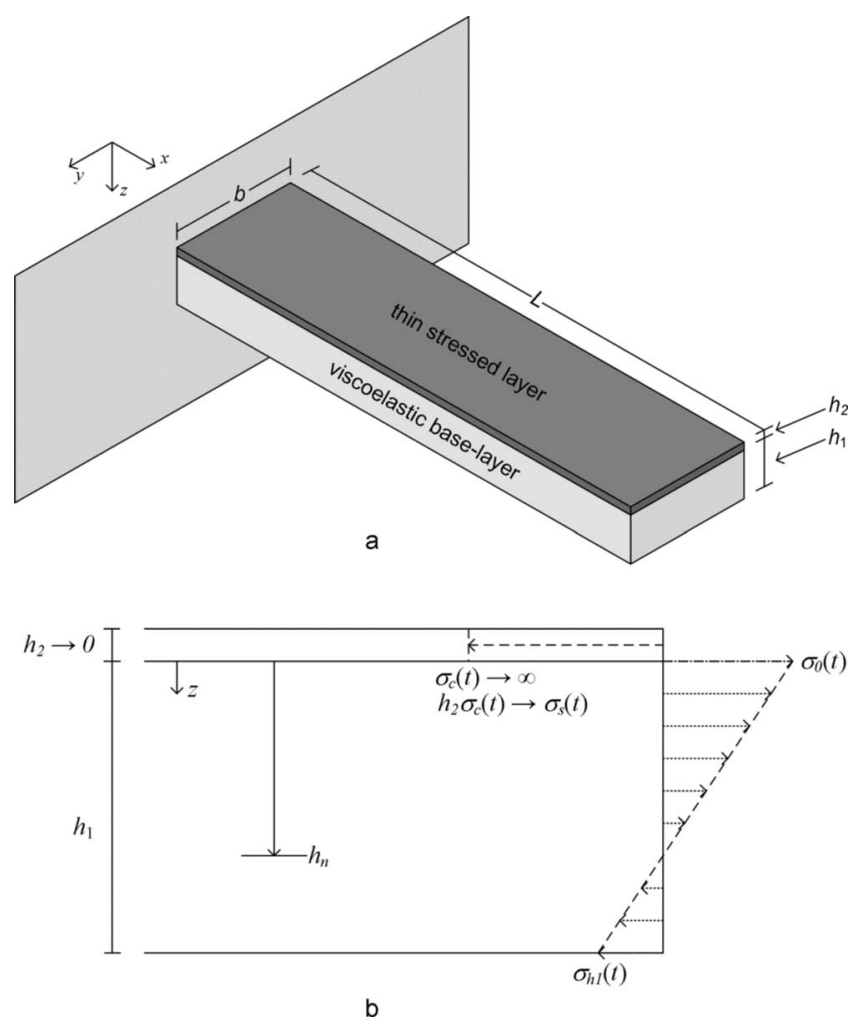

FIG. 1. (a) Illustration of the microcantilever geometry. (b) Stresses acting on a cross section of the microcantilever.

lever can result in a longer response time due to viscoelastic creep in the microcantilever. It may be possible to misinterpret creep in the microcantilever as continued adsorption. This, in turn, will lead to an overestimation of the analyte concentration if the time-dependence of the relaxation modulus is not considered. Using the generalized model, the above phenomena, which are overlooked when using Stoney's equation, can be taken into account so that it is possible to theoretically predict the microcantilever's bending response and choose polymer materials for which the response time is not increased. Furthermore, it is shown that the model can be used to extract the time-domain viscoelastic properties of the cantilever's material. These material properties are necessary to design devices and to predict sensor responses.

\section{THEORY}

\section{A. Formulation of the stress-curvature relationship}

Consider a viscoelastic beam as shown in Fig. 1(a), with length $L$, width $b$, and thickness $h_{1}$. A thin layer of thickness $h_{2} \ll h_{1}$ is mechanically bonded to the top of the cantilever beam. It is assumed that the beam is made from a rigid viscoelastic material (glassy polymer) that behaves as a three-parameter solid in terms of the biaxial stress/strain relationship. That is, in the cantilever, the biaxial stress and strain satisfy the three-parameter solid model,

$$
\sigma(t)+\tau_{r} \frac{d \sigma}{d t}=M_{\infty} \varepsilon(t)+\tau_{r} M_{0} \frac{d \varepsilon}{d t},
$$

where $M_{\infty}$ and $M_{0}$ are known as the asymptotic (relaxed) biaxial modulus and initial (unrelaxed) biaxial modulus, re-

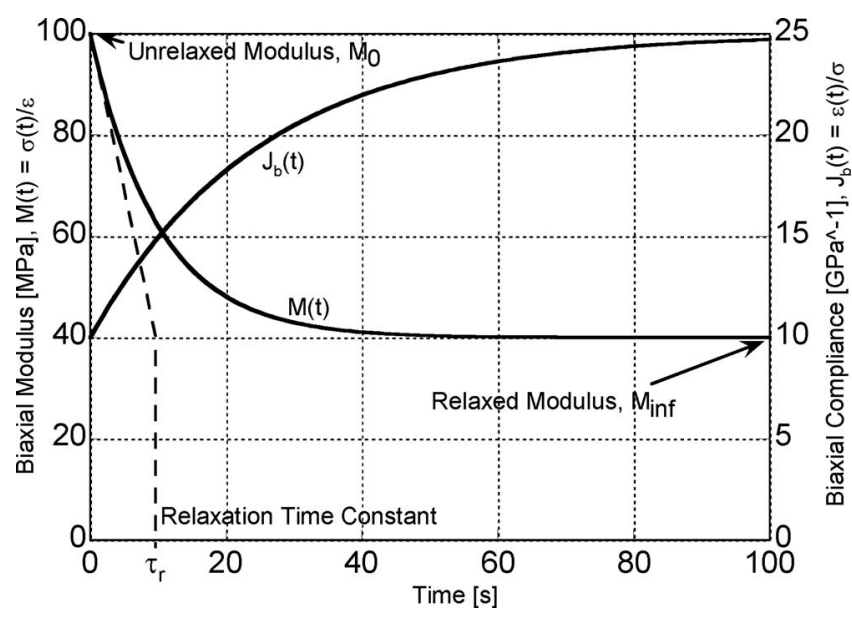

FIG. 2. Biaxial relaxation modulus, $M(t)$, (stress caused by a unit step strain) and the biaxial creep compliance, $J_{b}(t)$, (strain caused by a unit step stress) of a viscoelastic material modeled as a three-parameter solid.

spectively, and $\tau_{r}$ is the relaxation time constant. ${ }^{17}$ Thus, the beam material exhibits stress relaxation and creep (characteristics of most polymeric materials) as shown in Fig. 2. It is noted that a more complex viscoelastic stress/strain relationship could be used in place of the three-parameter solid model without much change in the procedure of the derivation. However, the three-parameter solid model is simple and exhibits the general characteristics of any viscoelastic material, namely, an initial modulus, an asymptotic modulus, and a parameter that describes the rate of the transition between the two moduli.

In the layer of thickness $h_{2}$, a uniform stress, $\sigma_{2}$, is assumed to exist. (This can be the result of various phenomena including residual stress of a deposited coating, a reduction in surface energy due to adsorption, repulsion among sorbed molecules, or thermal expansion of a thin coating.) It is assumed that $h_{2} \rightarrow 0$ and $\sigma_{2} \rightarrow \infty$ such that $h_{2} \sigma_{2} \rightarrow \sigma_{s}$, where $\sigma_{s}$ is known as the surface stress and has units $(\mathrm{N} / \mathrm{m})$ or force per beam width. The kinematic assumptions of elementary beam theory are invoked (Bernoulli-Euler and small defomations). ${ }^{18}$ This allows one to write the strain profile through the beam as a linear function that, by definition, is zero at the neutral axis,

$$
\varepsilon(z, t)=\kappa(t)\left[h_{n}(t)-z\right]
$$

where $\kappa$ is the curvature of the beam, $h_{n}$ defines the location of the neutral axis, and $z$ is the vertical coordinate taken positive downward from the top of the base layer, as shown in Fig. 1(b). Extensional strains and tensile stresses are taken positive. The curvature is defined as the second derivative of the deflection with respect to $x, \kappa=d^{2} w / d x^{2}$. Note that this notation implies that a positive curvature corresponds to downward deflection. The linear strain profile implies a linear stress profile [shown in Fig. 1(b)] by use of the hereditary integral representation of a viscoelastic material. ${ }^{12}$ Thus, it is possible to write the stress profile through the thickness of the cantilever in terms of the stress at the top $(z=0), \sigma_{0}(t)$, and at the bottom $\left(z=h_{1}\right), \sigma_{h 1}(t)$, of the cantilever, 


$$
\sigma(z, t)=\frac{\sigma_{h 1}(t)-\sigma_{0}(t)}{h_{1}} z+\sigma_{0}(t) .
$$

The structure (cantilever and thin upper layer) is not subjected to any external mechanical load. Thus, the resultant axial force and bending moment from the stress distribution and the surface stress must be zero (i.e., $\sigma_{s}(t)+\int_{0}^{h_{1}} \sigma(z, t) d z$ $=0$ and $\int_{0}^{h_{1}} z \sigma(z, t) d z=0$, where the moments are taken about the origin to eliminate contribution of the surface stress). Imposing these two conditions on the stress profile (4) results in the following two relations:

$$
\begin{aligned}
& \sigma_{0}(t)=-2 \sigma_{h 1}(t), \\
& \sigma_{0}(t)=-\frac{4}{h_{1}} \sigma_{s}(t) .
\end{aligned}
$$

Equations (4) and (5a) imply that at $z=(2 / 3) h_{1}$, the stress is always zero. This, in turn, implies that the neutral axis (i.e., the locus of points on the cross section for which the strain is zero) is given by $h_{n}=(2 / 3) h_{1}$. At any point through the thickness of the beam, the stress/strain relationship must be satisfied. Equation (2) may therefore be used to relate the stress and strain at the top $(z=0)$ of the cantilever,

$$
\sigma_{0}(t)+\tau_{r} \frac{d \sigma_{0}}{d t}=M_{\infty} \varepsilon_{0}(t)+\tau_{r} M_{0} \frac{d \varepsilon_{0}}{d t} .
$$

Substitution of Eqs. (3) and (5b) into Eq. (6) yields a differential equation relating the surface stress to the curvature of the beam as

$$
M_{\infty} h_{1}^{2} \kappa(t)+\tau_{r} M_{0} h_{1}^{2} \frac{d \kappa}{d t}=-6 \sigma_{s}(t)-6 \tau_{r} \frac{d \sigma_{s}}{d t} .
$$

The solution to this equation yields the curvature of the viscoelastic beam subjected to a specific applied surface stress history. It is noted that Eq. (7) reduces to Stoney's equation, with $M_{\infty}$ or $M_{0}$ in place of the biaxial modulus, as the relaxation time constant approaches zero or infinity, respectively. This is intuitive because, as the relaxation time constant approaches these limits, the time varying relaxation modulus becomes a constant and the material behaves as an elastic material. Similarly, if $M_{\infty} \approx M_{0}$ then the modulus again remains constant and the material behaves elastically. Note that there is a difference in sign between Stoney's Eq. (2) and Eq. (7). This arises because, in the present paper, a downward deflection is taken to be positive, whereas in Stoney's equation a downward deflection is negative.

\section{B. Application to polymer-based microcantilever sensors}

As previously stated, the stress generated at the surface of the cantilever can be from various sources. The physical phenomenon that is causing the surface stress will determine the time-dependent function $\sigma_{s}(t)$ (i.e., how the surface stress is applied with time). In the study of microcantilever sensors, the surface stress can be caused by molecules adsorbing onto the top surface of the cantilever. In the formulation of the problem, the adsorbed layer can be represented by the thin upper layer whose thickness, $h_{2}$, approaches zero. To deter- mine the adsorption-induced surface stress as a function of time, it is first necessary to determine how the number of adsorbed molecules is related to the ambient analyte concentration. This relationship is based on two assumptions. First, it is assumed that the number of adsorbed molecules at equilibrium is proportional to the ambient concentration of the analyte. Second, it is assumed that the rate of adsorption is proportional to the difference between the amount adsorbed at equilibrium and the current amount adsorbed. The constants of proportionality are the adsorption coefficient, $\eta$, and the adsorption decay rate, $\tau_{s}^{-1}\left(\tau_{s}\right.$ is the adsorption time constant), respectively. Under these assumptions the model for adsorption takes the form,

$$
\frac{d N}{d t}=\frac{1}{\tau_{s}}\left[\eta C_{\mathrm{amb}}(t)-N(t)\right],
$$

where $C_{\mathrm{amb}}(t)$ is the ambient concentration and $N(t)$ is the number of adsorbed analytes. For relatively small ambient concentrations causing single layer adsorption, this is a practical model as it represents type I adsorption. ${ }^{19}$

A surface stress is generated when adsorption of the analytes onto the surface of the microcantilever causes the analyte molecules to interact with each other and with the molecules that make up the adsorbing surface. ${ }^{20}$ For relatively small ambient concentrations causing low analyte coverage, the surface stress is primarily caused by the interaction between the adsorbate atoms and the adsorbing surface and is proportional to the analyte coverage. ${ }^{20}$ This stress can be obtained by analyzing the change in interfacial energy at the adsorption surface, using ${ }^{21}$

$$
\sigma_{s}=\gamma_{a, f}-\gamma_{a, i}=\Delta G_{a} \frac{\Gamma}{M},
$$

where $\gamma_{a, i}$ and $\gamma_{a, f}$ are the initial and final interfacial energies of the adsorbing surface, respectively, $\Delta G_{a}$ is the change in Gibb's free energy caused by adsorption, $\Gamma$ is the mass of absorbed analyte per unit area, and $M$ is the molar mass of the analyte. Thus, for a given surface area the surface stress is proportional to the number of adsorbed molecules, $\sigma_{s}(t)$ $=-\lambda N(t)$; then, Eqs. (7) and (8) imply that the overall system response of a viscoelastic cantilever undergoing adsorption is governed by

$$
\begin{aligned}
\frac{d \sigma_{s}}{d t}= & \frac{1}{\tau_{s}}\left[-\lambda \eta C_{\mathrm{amb}}(t)-\sigma_{s}(t)\right], \\
\frac{d \kappa}{d t}= & -\frac{1}{\tau_{r}} \frac{M_{\infty}}{M_{0}} \kappa(t)+\frac{6}{\tau_{r} M_{0} h_{1}^{2}}\left(\frac{\tau_{r}}{\tau_{s}}-1\right) \sigma_{s}(t) \\
& +\frac{6}{\tau_{s} M_{0} h_{1}^{2}} \lambda \eta C_{\mathrm{amb}}(t) .
\end{aligned}
$$

Note that the surface stress is compressive (negative) when it is caused by a reduction in the interfacial energy (a spontaneous adsorption process). ${ }^{21}$ Thus, $\lambda$ is generally positive.

Equations (10a) and (10b) can be solved for the case of a step-function ambient concentration, $C_{\mathrm{amb}}(t)=C_{\mathrm{amb}} u_{s}(t)$ [where $u_{s}(t)$ is the unit step function]. This would be equivalent to solving Eq. (7) for a surface stress given by $\sigma_{s}(t)=$ 
$-\lambda \eta C_{\mathrm{amb}}\left[1-\exp \left(-t / \tau_{s}\right)\right] u_{s}(t)$. It is noted that this form of the surface stress history (or adsorption kinetics) has been chosen as a practical representation of the adsorption processes of a hypothetical system. It is possible that the real adsorption kinetics (and the surface stress history) may be of a different form, depending on the specifics of the surface chemistry involved, and the ambient concentration associated with a particular analyte delivery system might not be accurately represented by a step function. In these cases, one can simply solve Eq. (7) with the appropriate surface stress history, obtained from the adsorption kinetics and the specific analyte delivery function [rather than $\left.u_{s}(t)\right]$.

The analytical solution to Eqs. (10a) and (10b) for a step in ambient concentration is given by

$$
\begin{aligned}
\kappa(t)= & -\frac{6 \sigma_{s s}}{M_{\infty} h_{1}^{2}}\left\{1+\frac{(\bar{\tau}-1) \bar{M}}{1-\bar{\tau} \bar{M}} \exp \left[-\frac{t}{\tau_{s}}\right]\right. \\
& \left.-\frac{1-\bar{M}}{1-\bar{\tau} \bar{M}} \exp \left[-\bar{M} \frac{t}{\tau_{r}}\right]\right\} u_{s}(t),
\end{aligned}
$$

where $\sigma_{s s}=-\lambda \eta C_{\mathrm{amb}}$ is the steady-state surface stress and the following dimensionless parameters have been introduced:

$$
\begin{aligned}
& \bar{M}=\frac{M_{\infty}}{M_{0}}, \\
& \bar{\tau}=\frac{\tau_{s}}{\tau_{r}} .
\end{aligned}
$$

In the case where the eigenvalues of the differential equations in Eqs. (10a) and (10b) are repeated, i.e., when $\bar{\tau} \bar{M}$ $=1$, Eq. (11) does not hold. This occurs when the creep time constant, $\tau_{c} \equiv\left(M_{0} / M_{\infty}\right) \tau_{r}$, and the adsorption time constant are identical. In order to find the tip deflection, one simply integrates the constant curvature twice to obtain $w_{\text {tip }}$ $=\kappa\left(L^{2} / 2\right)$.

\section{Coated microcantilever sensors}

In practical devices, adsorption of analytes onto the microcantilever is usually facilitated by a coating (thin rubbery polymer or metal), which has some affinity for the analyte. In this case, the coating, which is assumed to be initially unstressed, is represented by a third layer that is between the microcantilever, $h_{1}$, and the thin layer of adsorbed molecules, as shown in Fig. 3. To use the above equations to analyze such (bio)chemical sensors, the coating must be thin and soft so that any stresses in the coating caused by bending of the microcantilever do not have a significant effect on the axial force or the bending moment. These stresses in the coating are usually neglected in the case of an elastic (e.g., silicon) cantilever because the modulus of the cantilever material is quite large. Therefore, the stresses in the cantilever dominate the stresses in the coating for a wide range of coating moduli and thicknesses. This is likely to still be true when the viscoelastic cantilever is coated with a polymer. However, even a thin metal coating may add significant rigidity to the beam. As a result, Eq. (11) is valid for metal coated cantilevers only if the metal thickness is to be less than about $1 \%-3 \%$ the

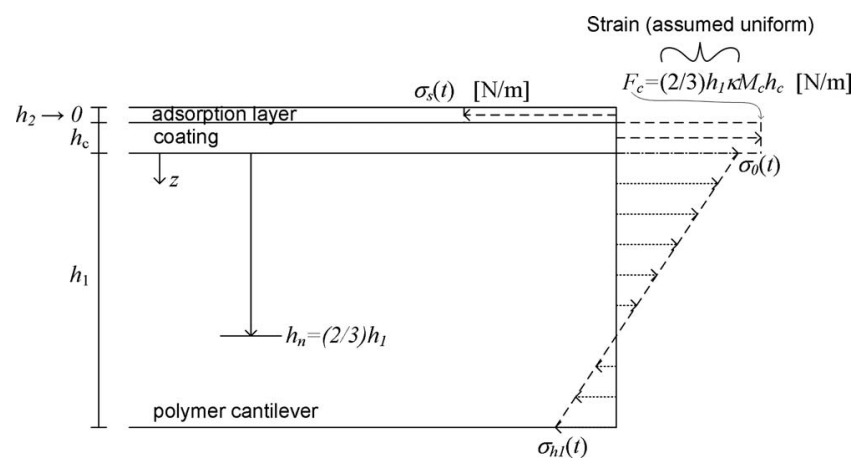

FIG. 3. Schematic of a coated microcantilever sensor with an adsorption layer. The stresses acting on a cross section of the microcantilever beam are illustrated.

cantilever's thickness depending on the modulus of the cantilever and of the metal coating. More specifically, as will be shown, it is required that $M_{\infty} h_{1} \gg M_{c} h_{c}$, where $M_{c}$ and $h_{c}$ are the biaxial modulus and thickness of the thin elastic coating, respectively. If this condition is not met, the governing Eq. (7) can be modified to account for the stress in the coating.

For a thin and elastic coating, the stress resulting from the coating being extended during bending can be treated as a concentrated force per unit width acting at the interface, $z=0$, along with the surface stress. As a result, Eq. (5a) and the location of the neutral axis remain the same as in the previous derivation and the resultant axial force caused by the stress in the coating can be approximated as $F_{c}$ $=(2 / 3) h_{1} \kappa M_{c} h_{c}$. Requiring that the resultant axial force through a cross section of the beam be zero, one obtains

$$
\sigma_{0}(t)=-\frac{4}{h_{1}} \sigma_{s}(t)-\frac{8}{3} M_{c} h_{c} \kappa(t) .
$$

Substituting Eqs. (3) and (13) into Eq. (6), the governing equation relating the curvature to the surface stress when the stress in the coating is significant can be written as

$$
\begin{aligned}
& \left(M_{\infty}+4 \frac{h_{c}}{h_{1}} M_{c}\right) h_{1}^{2} \kappa(t)+\tau_{r}\left(M_{0}+4 \frac{h_{c}}{h_{1}} M_{c}\right) h_{1}^{2} \frac{d \kappa}{d t} \\
& =-6 \sigma_{s}(t)-6 \tau_{r} \frac{d \sigma_{s}}{d t} .
\end{aligned}
$$

Equations (14) and (7) have the same form. Therefore, the analytical solution to Eq. (7) can still be used by defining an effective initial biaxial modulus and an effective asymptotic biaxial modulus for the coated beam as

$$
\begin{aligned}
& M_{0, \mathrm{eff}}=\left(M_{0}+4 \frac{h_{c}}{h_{1}} M_{c}\right), \\
& M_{\infty, \mathrm{eff}}=\left(M_{\infty}+4 \frac{h_{c}}{h_{1}} M_{c}\right),
\end{aligned}
$$

respectively. The structural behavior of the coated cantilever is identical to a homogeneous beam made from a three parameter solid having the effective moduli as defined in Eqs. (15a) and (15b) and the original relaxation time constant as the material property. 
Even a very thin metal coating will significantly raise the effective modulus of the coated beam. For example, a $50 \mathrm{~nm}$ gold coating with a biaxial modulus of $M_{c}=95 \mathrm{GPa}$, bonded to a $2 \mu \mathrm{m}$ SU-8 cantilever $\left(M_{0}=4.5 \mathrm{GPa}, M_{\infty}=3.7 \mathrm{GPa}\right)$ has an effective initial biaxial modulus of $14 \mathrm{GPa}$ and an effective asymptotic modulus of $13.2 \mathrm{GPa}$. The coated beam "effectively" relaxes by only $6 \%$ (compared with $18 \%$ relaxation for the uncoated SU-8) and could thus be approximated quite well as an elastic beam. However, this assumes that such thin gold films are themselves elastic. Some thin metal films have been known to exhibit viscoelastic behavior. ${ }^{22}$

If the stress in the coating due to bending of the microcantilever is significant and the coating can be modeled as a viscoelastic three-parameter solid, it is still possible to define similar effective biaxial moduli under certain conditions. Assuming the resultant force in the coating acts at the interface $(z=0)$, the neutral axis remains the same as in the previous derivations, $h_{n}=(2 / 3) h_{1}$. Thus, because the coating is thin, the strain in the coating can be assumed uniform and approximated as $\varepsilon_{c}=(2 / 3) h_{1} \kappa$. Requiring the total axial force to be zero, one obtains two coupled differential equations describing the curvature of a viscoelastic cantilever coated with a thin viscoelastic material under an applied surface stress as

$$
\begin{aligned}
& 2 M_{\infty, c} h_{1} \kappa(t)+2 \tau_{r, c} M_{0, c} h_{1} \frac{d \kappa}{d t}=3 \sigma_{c}(t)+3 \tau_{r, c} \frac{d \sigma_{c}}{d t}, \\
& M_{\infty} h_{1}^{2} \kappa(t)+\tau_{r} M_{0} h_{1}^{2} \frac{d \kappa}{d t}+6 h_{c} \sigma_{c}(t)+6 \tau_{r} h_{c} \frac{d \sigma_{c}}{d t} \\
& =-6 \sigma_{s}(t)-6 \tau_{r} \frac{d \sigma_{s}}{d t},
\end{aligned}
$$

where $\sigma_{c}$ is the stress in the coating. These equations can be further simplified to fit the form of Eq. (14) if the relaxation time constant of the coating is the same as that of the cantilever material,

$$
\begin{aligned}
& \left(M_{\infty}+4 \frac{h_{c}}{h_{1}} M_{\infty, c}\right) h_{1}^{2} \kappa(t)+\tau_{r}\left(M_{0}+4 \frac{h_{c}}{h_{1}} M_{0, c}\right) h_{1}^{2} \frac{d \kappa}{d t} \\
& =-6 \sigma_{s}(t)-6 \tau_{r} \frac{d \sigma_{s}}{d t} .
\end{aligned}
$$

Equations (17) and (7) have the same form. Therefore, the structural behavior of the composite beam (viscoelastic coating and a viscoelastic base layer) is identical to a homogeneous beam made from a three parameter solid having the effective moduli,

$$
\begin{aligned}
& M_{0, \mathrm{eff}}=\left(M_{0}+4 \frac{h_{c}}{h_{1}} M_{0, c}\right), \\
& M_{\infty, \mathrm{eff}}=\left(M_{\infty}+4 \frac{h_{c}}{h_{1}} M_{\infty, c}\right) .
\end{aligned}
$$

Note that if the relaxation time constant of the coating is not similar to that of the cantilever material then the composite (coated beam) will not behave as a three-parameter solid and this definition of effective moduli does not hold. In this case,
Eqs. (16a) and (16b) can still be applied to obtain the deflection of the cantilever as a function of time.

As an example of a polymer microcantilever coated with a viscoelastic material, consider a $2 \mu \mathrm{m}$ SU-8 cantilever with $50 \mathrm{~nm}$ aluminum coating. A $1.16 \mu \mathrm{m}$ thick aluminum film was shown to exhibit viscoelastic behavior. ${ }^{22}$ By fitting the experimental data in that work with a three-parameter solid model the $1.16 \mu \mathrm{m}$ thick aluminum was found to have an initial Young's modulus of $70 \mathrm{GPa}$ and an asymptotic modulus of $60 \mathrm{GPa}$ with a relaxation time constant of roughly $600 \mathrm{~s}$. Using a constant Poisson's ratio of 0.35 , the corresponding values of the initial and asymptotic biaxial modulus of the thin aluminum coating are 108 and $92 \mathrm{GPa}$, respectively. A $2 \mu \mathrm{m}$ SU-8 cantilever with a $50 \mathrm{~nm}$ aluminum coating would have effective initial and asymptotic biaxial moduli of 15.3 and $12.9 \mathrm{GPa}$, respectively, using Eqs. (18a) and (18b) (assuming that the properties of a $50 \mathrm{~nm}$ thick aluminum are the same as those of a $1.16 \mu \mathrm{m}$ thick aluminum). In this case, the relaxation factor is nearly unchanged and the overall response is simply a scaled version of the response obtained from Eq. (7), not taking into account the stresses in the coating. Here, the initial biaxial modulus of the coating was used to modify the initial biaxial modulus of the polymer cantilever, and the asymptotic biaxial modulus of the coating was used to modify the asymptotic biaxial modulus of the cantilever.

\section{RESULTS AND DISCUSSION}

This section will be organized into three subsections. The first will examine how the response depends on the relaxation factor $(1-\bar{M})$ and the time constant ratio $(\bar{\tau})$. Then, the benefits and consequences of using a microcantilever sensor made from a viscoelastic material will be discussed. Finally, a simple method for extracting the time-domain viscoelastic properties of the cantilever material will be proposed.

A polymer that is receiving much attention for the implementation of microcantilevers is SU-8. Previous studies have shown that it is feasible to manufacture and implement microcantilever (bio)chemical sensors from SU-8. ${ }^{16}$ Therefore, many of the calculations and simulations in this paper will use the material properties of SU-8 for the polymeric microcantilever. The viscoelastic properties of SU-8 have been previously characterized in terms of the (uniaxial) relaxation modulus. ${ }^{23}$ Fitting this relaxation modulus to the threeparameter solid model one obtains an initial modulus in the range of 3.1-3.2 GPa, an asymptotic modulus in the range of 2.5-2.7 GPa, and a relaxation time constant of approximately 600 s. The Poisson's ratio of SU-8 is approximately $0.3 .^{24}$ Using this data to approximate the biaxial behavior, one obtains initial and asymptotic biaxial moduli of 4.5 and 3.7 $\mathrm{GPa}$, respectively, and the same relaxation time constant (600 s).

\section{A. Cantilever response}

The analytical solution [Eq. (11)] for the cantilever bending due to an applied surface stress is plotted in Fig. 4. Normalized curvature $\left(\kappa M_{\infty} h_{1}^{2} / 6 \sigma_{s s}\right)$ or normalized deflec- 


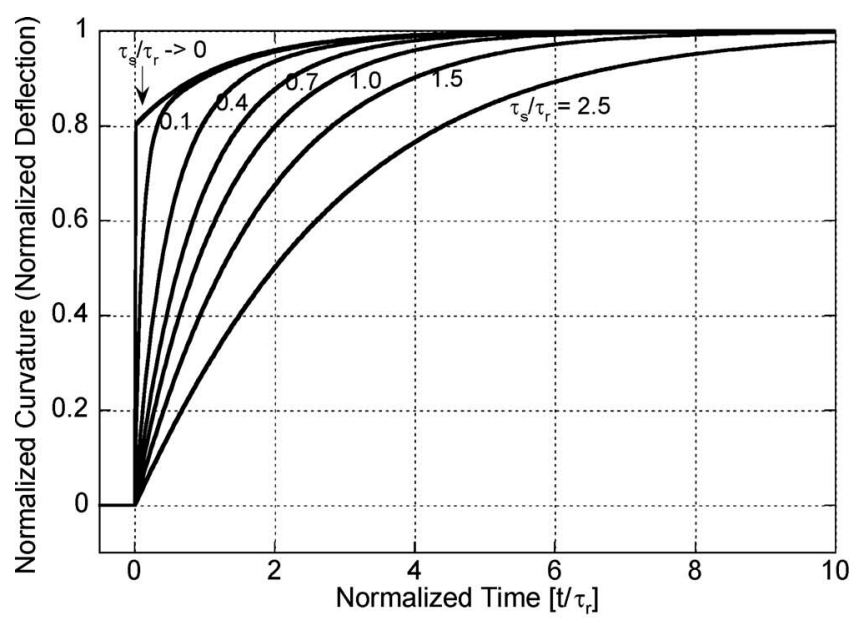

a

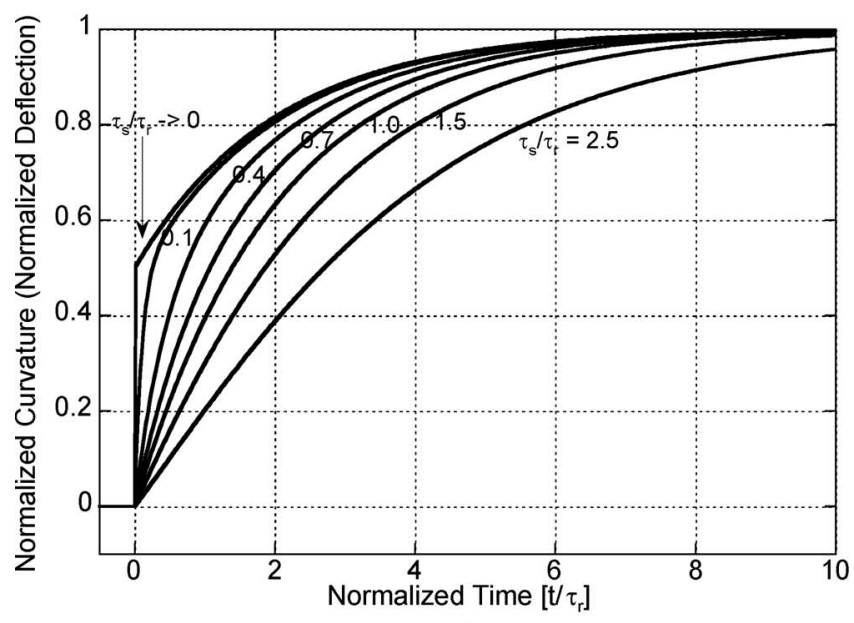

b

FIG. 4. Theoretical bending responses of a polymeric microcantilever for different analyte/coating pairs represented by varying $\tau_{s} / \tau_{r}$. (a) Polymer relaxation factor $=0.2$. (b) Polymer relaxation factor $=0.5$.

tion $\left(w_{\text {tip }} M_{\infty} h_{1}^{2} / 3 L^{2} \sigma_{s s}\right)$ is the ordinate while normalized time $\left(t / \tau_{r}\right)$ is the abscissa. This forces the steady-state value of the normalized curvature (and deflection) to be one. A family of curves is shown for two different relaxation factors (1 $-M_{\infty} / M_{0}=0.20$ and 0.50$)$. For each relaxation factor the response is shown for several time constant ratios $\left(\tau_{s} / \tau_{r}\right): 0$, $0.1,0.4,0.7,1.0,1.5$, and 2.5. Similarly, in Fig. 5, the normalized deformation is shown as a function of time, where the time is now normalized by the sorption time constant $\left(t / \tau_{s}\right)$. This normalization has the effect of showing the family of responses for a fixed sorption time constant, whereas Fig. 4 shows the responses for a fixed relaxation time constant. Thus, one could examine Fig. 4 to investigate the possible responses that a specific microcantilever sensor (particular values of relaxation factor and relaxation time constant) will have to various analyte/thin coating pairs (various adsorption dynamics). Alternatively, Fig. 5 can be used to analyze the possible responses that a specific analyte/ thin coating pair (a particular adsorption dynamic) would exhibit when used on different polymeric cantilevers (various relaxation factors and relaxation time constants). The effects of the polymer base layer and the coating have been

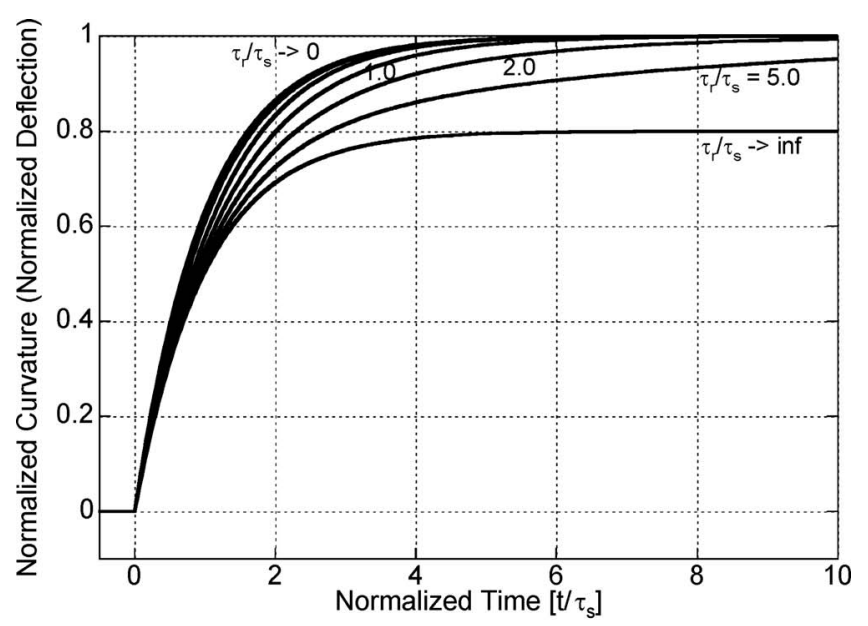

a

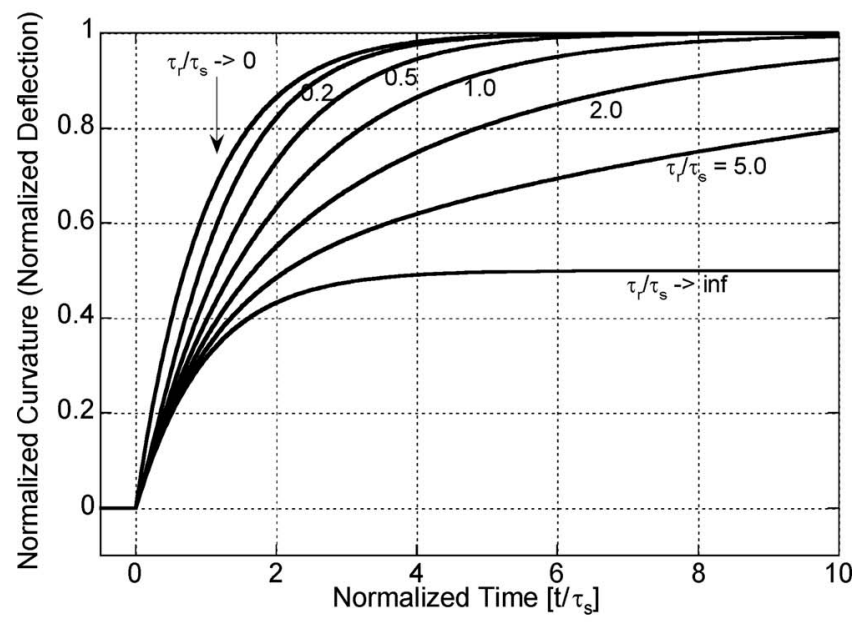

b

FIG. 5. Theoretical bending responses of different polymeric microcantilevers with the same analyte/coating pair. The different microcantilevers are represented by varying $\tau_{r} / \tau_{s}$. (a) Polymer relaxation factor $=0.2$. (b) Polymer relaxation factor $=0.5$.

separated because it is assumed that the base layer is chemically resistive and thus does not affect adsorption or surface stress generation and that the coating is thin enough that it does not affect the effective moduli, as defined in the previous section. It is noted these types of responses exhibiting creep have been observed using SU-8 cantilever. ${ }^{16}$

Figure 5 illustrates some important differences between the response of an elastic cantilever and that of a polymeric cantilever. Stoney's Eq. (1) indicates that, for an elastic cantilever, the deflection and the surface stress are proportional. For a time-varying surface stress, Stoney's equation holds pointwise in time and the deflection history is a scaled version of the surface stress history. Therefore, the response of an elastic cantilever is governed by only the adsorption time constant (i.e., the time constant of the surface stress, $\tau_{s}$ ). On the other hand, Stoney's equation, with $M_{0}$ or $M_{\infty}$ as the biaxial modulus of the base layer, only provides the lower or upper bounds for the response of a polymeric cantilever. At the limits $\tau_{r} / \tau_{s} \rightarrow \infty$ or $\tau_{r} / \tau_{s} \rightarrow 0$, the polymeric cantilever behaves as an elastic material because the material either never relaxes or is completely relaxed, respectively, during 
the time span of interest. However, for an arbitrary value of $\tau_{r} / \tau_{s}$, the response starts in the neighborhood of the lower bound curve and transitions toward the upper bound curve at a rate dependent on the ratio of the time constants. Note that the response does not reach the steady state until surface adsorption has reached equilibrium and the creep process in the base layer has ended. This can lead to a much longer response time than that predicted by Stoney's equation for an elastic beam.

\section{B. Sensitivity and response time}

Two of the most important characteristics of a chemical sensor are its sensitivity and its response time. As previously mentioned, creep in the microcantilever can continue long after adsorption is complete. This means that the polymeric microcantilever takes longer to respond than an elastic (silicon, for example) microcantilever with the same coating. Most importantly, if one is not aware of creep occurring in the microcantilever, it is possible to confuse the creep with continued adsorption, which will lead to a poor understanding of the adsorption dynamics and possibly an overestimation of the actual analyte concentration. However, sensitivity (defined as $w_{\text {tip }} / \sigma_{s s}$ ) is greatly improved when using a polymeric microcantilever due to the fact that most polymers have a much smaller biaxial modulus than that of typical elastic microcantilevers (e.g., silicon, which has a biaxial modulus of approximately $200 \mathrm{GPa}$ ). Thus, one gains sensitivity at the cost of an increase in response time. However, it is possible to minimize this increase in response time through careful polymer selection.

Both optical and piezoresistive techniques have been successfully used as readout methods for polymer-based microcantilevers. ${ }^{16}$ The results presented in this section are independent of the readout method employed as long as the output is proportional to the tip deflection. This is the case for optical or piezoresistive readout schemes. However, in the case of a piezoresistive readout scheme, the piezoresistive element may contribute significantly to the stiffness of the beam and may need to be treated as a coating, as described in Sec. II C.

Because creep will often occur after adsorption has reached equilibrium, standard definitions of response time may not be appropriate to characterize the response time of a polymeric microcantilever. In chemical sensor applications, any definition of the response time should be correlated with the time required to make an accurate estimate of the analyte concentration. Often, the steady-state sensor response is used to determine the analyte concentration. Thus, the time required to reach $90 \%$ of the steady-state response is commonly defined as the response time. This definition of response time, if applied to polymeric cantilevers, may not relate to the time required to make an accurate estimation of analyte concentration. Cantilevers with a large relaxation time constant exhibit very slow creep and take an extremely long time to reach the steady-state response. However, if creep occurs at a much slower rate than that of absorption, the response of a polymeric cantilever will be similar to that of an elastic cantilever. In that case, it is not necessary to
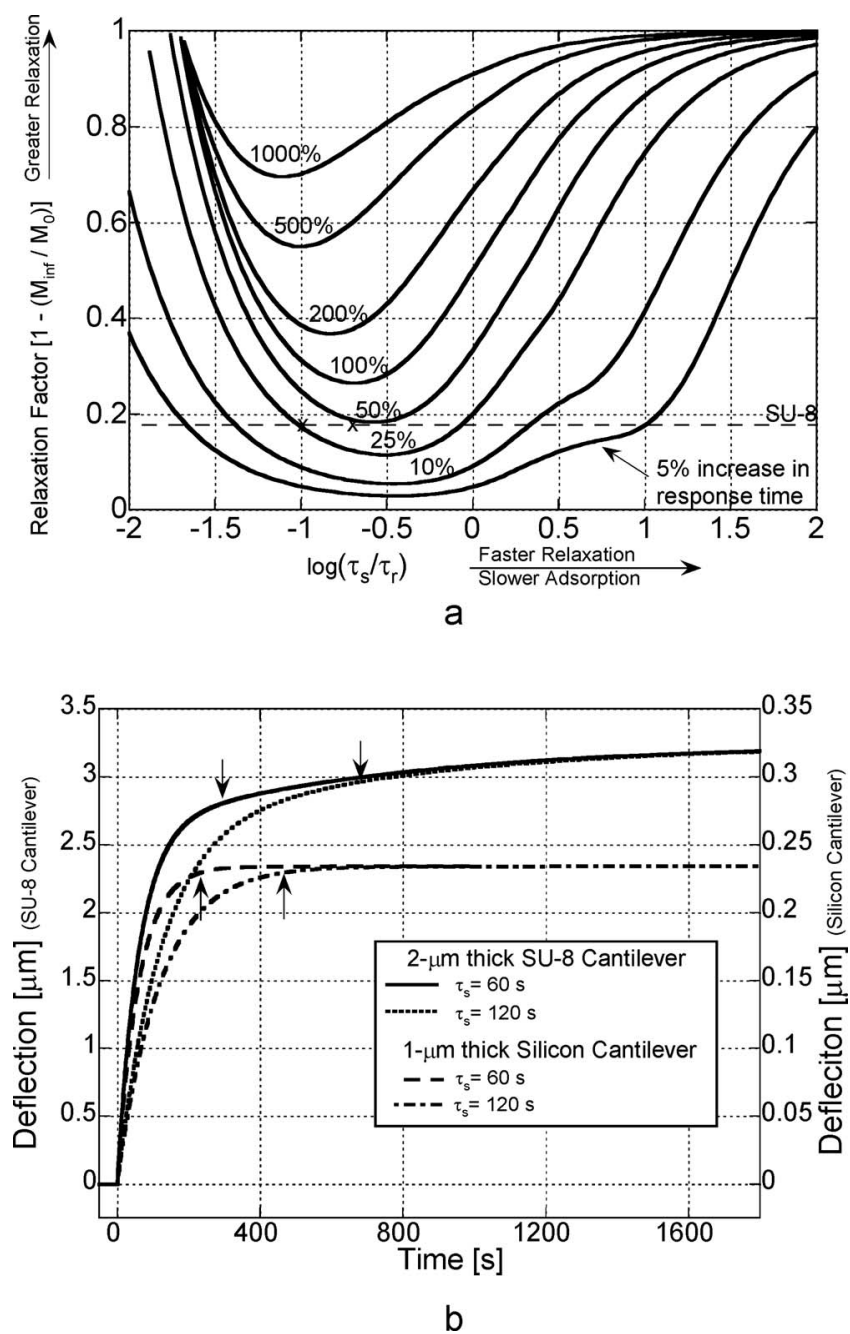

FIG. 6. (a) Loci of increase in response time of a polymeric cantilever relative to that of an elastic cantilever. The increase in response time is dependent only on the relaxation factor of the polymer and the time constant ratio. (b) Bending response of an SU-8 microcantilever (length $=400 \mu \mathrm{m}$, thickness $=2 \mu \mathrm{m}$ ) and silicon microcantilever (length $=400 \mu \mathrm{m}$, thickness $=1 \mu \mathrm{m})$ to analyte adsorption resulting in a steady-state surface stress of $-0.1 \mathrm{~N} / \mathrm{m}$ and having adsorption time constants of 60 and $120 \mathrm{~s}$. The arrows represent the sensor response times. Material properties determined from Refs. 23 and $24, M_{\infty}=4.5 \mathrm{GPa}, M_{0}=3.7 \mathrm{GPa}, \tau_{r}=600 \mathrm{~s}$, were used to represent SU-8.

wait for the sensor to creep to its steady state in order to obtain an estimate of the analyte concentration. After adsorption has reached equilibrium, the slope of the response is small enough that a final measurement can be taken at any time and used to estimate the analyte concentration without much variation. The fact that the sensor has not reached its steady state (after creep) is not an issue because the sensor would be calibrated using experimental runs stopped at approximately the same time. An appropriate definition of response time is therefore linked to the slowing of the response with time. As a result, response time may be defined here as the time at which the slope of the response has decayed to $2 \%$ its maximum slope. (Of course, other definitions are possible.) For elastic cantilevers that undergo adsorption governed by Eq. (8), the response time is just under four sorption time constants $\left[t_{r}=3.912 \tau_{s}\right.$, see Fig. 6(b)]. However, for 
polymeric cantilevers the response time is governed by the adsorption time constant and the material properties of the polymer.

The normalized response time (i.e., response time divided by the adsorption time constant) can be calculated from Eq. (11) and is a function of the relaxation factor and of the time constant ratio $\left(\tau_{s} / \tau_{r}\right)$. Loci of increase in response time for a polymeric cantilever relative to that of an elastic (silicon, for example) cantilever are shown in Fig. 6(a). For a given relaxation factor, the largest increase in response time occurs when the relaxation time constant is roughly two to ten times greater than the adsorption time constant. Also, for a given time constant ratio, $\tau_{s} / \tau_{r}$, the largest increase in response time occurs for large relaxation factors. The results in Fig. 6(a) also allow one to develop guidelines for the selection and use of polymer cantilevers. For example, if one was to limit the allowable increase in response time to $25 \%$, SU-8, which has a relaxation factor of 0.18 and a relaxation time constant of $600 \mathrm{~s}$, could be used to detect adsorption processes with a time constant of less than $60 \mathrm{~s}$ or more than 600 s. Figure 6(a) also indicates that an SU-8 microcantilever could be used for all adsorption processes (i.e., an arbitrary value of the adsorption time constant) if one was willing to accept response times that were no more than 50\% larger than the response time of an elastic cantilever. Figure 6(b) shows the simulated responses of a $2 \mu \mathrm{m}$ thick SU-8 microcantilever and a $1 \mu \mathrm{m}$ thick silicon microcantilever detecting surface adsorption with a time constant of $60 \mathrm{~s}$ leading to an equilibrium surface stress of $-0.1 \mathrm{~N} / \mathrm{m}$. (Results for an adsorption time constant of $120 \mathrm{~s}$ are also shown.) These responses for SU-8 are represented by the $x$ on Fig. 6(a). In the case of a $60 \mathrm{~s}$ adsorption time constant, the response time [represented by arrows in Fig. 6(b)] is 235 s for an elastic cantilever and 296 s for a SU-8 cantilever, which represents an increase of $26 \%$. When the adsorption time constant is increased to $120 \mathrm{~s}$, the response time is 469 $\mathrm{s}$ for an elastic cantilever and $682 \mathrm{~s}$ for a SU-8 cantilever, which represents an increase of $45 \%$. It is reiterated that the specific values for the response times calculated in this section are based on the definition of response time as the time at which the slope of the response has decayed to $2 \%$ its maximum value.

Equation (11) indicates that the sensitivity of the polymer cantilever is inversely proportional to $M_{\infty} h_{1}^{2}$. Thus, the sensitivity of a polymer cantilever is increased greatly compared to that of a silicon cantilever. However, it is also necessary to consider the thickness of the polymeric cantilever because each polymer may have a different fabrication limit for the smallest possible thickness of the cantilever. Silicon cantilevers can be produced with thicknesses less than $0.5 \mu \mathrm{m}$, whereas reported SU-8 cantilevers have thicknesses of $2 \mu \mathrm{m}$. However, even when the polymer cantilever is thicker, in most cases, sensitivity is still increased because the modulus is much smaller. Figure 6(b) shows that the sensitivity of the $2 \mu \mathrm{m}$ thick SU-8 cantilever is 13.9 times greater than that of the $1 \mu \mathrm{m}$ thick silicon cantilever.

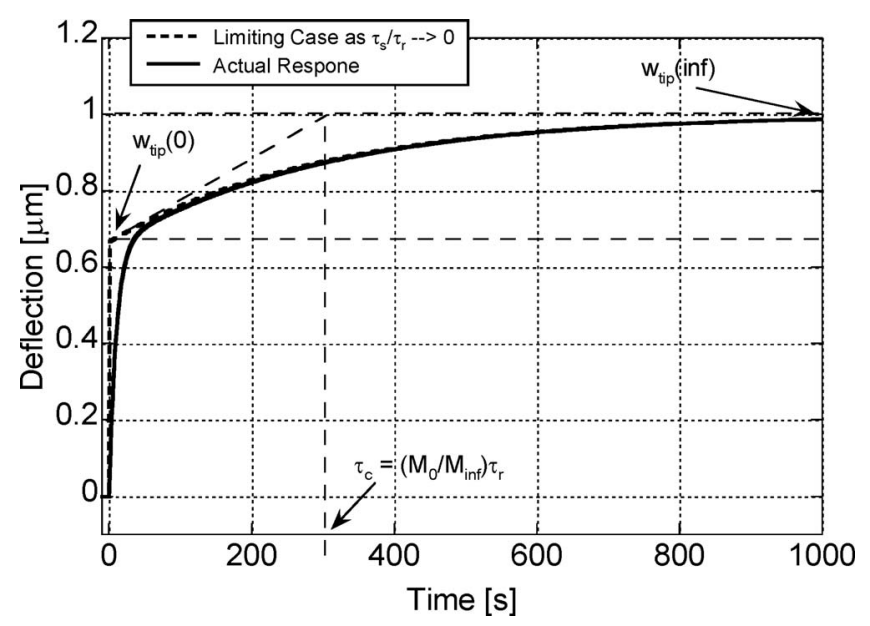

FIG. 7. Determination of the initial and asymptotic cantilever deflection and the creep time constant from the bending response of the polymeric cantilever. These parameters are used for the extraction of the time-domain viscoelastic properties of the cantilever. The cantilever exhibits $33 \%$ relaxation and a creep time constant of $300 \mathrm{~s}$.

\section{Extraction of viscoelastic properties}

Some polymers have been characterized in the frequency domain at a specific frequency ${ }^{25,26}$ or for a range of frequencies. ${ }^{27}$ However, few data on the time-domain viscoelastic properties of polymer materials are available in the literature; examples of two exceptions are polyisobutylene and recently SU-8. ${ }^{23,27}$ Also, it is noted that the polymer material properties may depend on fabrication processes such as curing, deposition technique, etc. The theory presented herein can be used to provide a simple technique to extract the time-domain viscoelastic properties of a polymer from the cantilever's response to a surface stress.

If a surface stress is applied to the microcantilever very rapidly with respect to the relaxation time constant, then $\bar{\tau}$ approaches zero. In this limit, Eq. (11) can be simplified and the beam curvature can be written as

$$
\kappa(t)=-\frac{6 \sigma_{s s}}{h_{1}^{2} M_{\infty}}\left[1-\left(1-\frac{M_{\infty}}{M_{0}}\right) \exp \left(-\frac{M_{\infty}}{M_{0}} \frac{t}{\tau_{r}}\right)\right] u_{s}(t) .
$$

Equation (19), which has the same form as the biaxial creep compliance, $J_{b}(t)$, for a three-parameter solid (Fig. 2), indicates that the cantilever's deflection at time, $t=0$, is given by

$$
w_{\text {tip }}(0)=-\frac{3 L^{2} \sigma_{s s}}{h_{1}^{2} M_{0}},
$$

and that the steady-state deflection is given by

$$
w_{\text {tip }}(\infty)=-\frac{3 L^{2} \sigma_{s s}}{h_{1}^{2} M_{\infty}} .
$$

The time constant of the transition between the initial and steady-state deflection is the creep time constant, defined as ${ }^{28}$

$$
\tau_{c} \equiv \frac{M_{0}}{M_{\infty}} \tau_{r}
$$

The values of $w_{\text {tip }}(0), w_{\text {tip }}(\infty)$, and $\tau_{c}$ in Eqs. (20)-(22) can be determined graphically, as shown in Fig. 7, when sorption is very fast compared to the relaxation. If the geometry and the 
steady-state applied surface stress $\left(\sigma_{s s}\right)$ are known, the equations can then be used to extract $M_{0}, M_{\infty}$, and $\tau_{r}$ directly from the cantilever response. These parameters completely characterize the polymer material for a three-parameter solid. However, if the geometry and/or the steady-state applied surface stress are unknown, it is still possible to extract the relaxation factor and the relaxation time constant by dividing Eq. (21) into Eq. (20) to obtain

$$
\frac{M_{\infty}}{M_{0}}=\frac{w_{\text {tip }}(0)}{w_{\text {tip }}(\infty)},
$$

and using Eq. (22) to determine the relaxation time constant. These two parameters, the relaxation factor and relaxation time constant, are sufficient to determine the response time of the polymeric microcantilever.

It is noted that the surface stress could be induced on the cantilever by several methods. Bonding a very thin, elastic material to the microcantilever and imposing a small thermal load or fast chemical adsorption are two possible techniques to apply the rapid surface stress. If the surface stress can be generated very rapidly compared to the relaxation time constant (for example, $\tau_{s}<0.05 \tau_{r}$ ), it will be possible to measure the initial and steady-state deflections and the creep time constant directly from the response. As shown in Fig. 7, this can be done by estimating the limiting case of the response given by Eq. (19). Equations (20)-(23) can then be used to obtain the material properties of the polymer. If the surface stress is induced slowly (compared to the previous example), the extraction of polymer properties may still be possible by fitting the measured response to Eq. (11). Again, the geometry and the steady-state elongation must be known in order to extract all material properties and the sorption time constant. Otherwise, one can obtain the relaxation factor, the relaxation time constant, and the sorption time constant. If relaxation occurs much faster than sorption, it may not be possible to extract any information about the initial modulus or the relaxation time constant. When relaxation occurs rapidly compared to sorption, the differences between the response of a polymeric microcantilever and that of an elastic microcantilever (with biaxial modulus equal to the asymptotic modulus of the polymeric cantilever) will be so small that the material properties cannot be extracted using the presented technique.

\section{CONCLUSION}

A theory of the bending of a polymeric (viscoelastic) microcantilever undergoing adsorption-induced surface stress is presented. The theory includes the effects of viscoelastic creep in the polymeric cantilever and the solution to the governing equation shows that, in many cases, creep in the microcantilever would cause a microcantilever-based sensor to continue to respond after adsorption is complete, thereby leading to a longer response time than that of an elastic (e.g., silicon) microcantilever. The response time can be doubled or even tripled in some cases. In chemical sensor applications, creep exhibited in the sensor response would, in turn, lead to an increase in the time of detection of the target analyte and would lead to a misunderstanding of adsorption dynamics and an overestimation of analyte concentration if viscoelastic creep is not taken into account in the analysis. However, many polymers, for which creep is not as prominent, could be used to develop microcantilever sensors. For example, using the developed theory, it is shown that an SU-8 microcantilever would, in the worst case, have a response time that is $50 \%$ longer than that of a silicon cantilever, but with an increase in sensitivity by more than a factor of 10. Thus, SU-8 appears to be a viable microcantilever material provided that viscoelastic creep is accounted for in the analysis.

A simple technique that can be used to extract the timedomain viscoelastic properties of the microcantilever is also proposed. This technique will be helpful in the characterization, and the selection, of glassy polymers that show potential for the implementation as microcantilever-based sensors. Knowledge of material properties will be used to better understand the device response. Furthermore, with this information it will be possible to predict the sensor's steady-state response and extract the adsorption dynamics from the sensor response.

\section{ACKNOWLEDGMENTS}

The authors would like to thank Professor Isabelle Dufour for helpful discussion.

${ }^{1}$ L. A. Pinnaduwage, H. Ji, and T. Thundat, IEEE Sens. J. 5, 774 (2005).

${ }^{2}$ T. Thundat, R. J. Warmack, G. Y. Chen, and D. P. Allison, Appl. Phys. Lett. 64, 2894 (1994).

${ }^{3}$ T. Thundat, E. A. Wachter, S. L. Sharp, and R. J. Warmack, Appl. Phys. Lett. 66, 1695 (1995).

${ }^{4}$ G. Y. Chen, T. Thundat, E. A. Wachter, and R. J. Warmack, J. Appl. Phys. 77, 3618 (1995)

${ }^{5}$ H. F. Ji, E. Finot, R. Dabestani, T. Thundat, G. M. Brown, and P. F. Britt, Chem. Commun. (Cambridge) 2000457.

${ }^{6}$ G. Y. Chen, T. Thundat, E. A. Wachter, and R. J. Warmack, J. Appl. Phys. 77, 3618 (1995).

${ }^{7}$ N. V. Lavrik, M. J. Sepaniak, and P. G. Datskos, Rev. Sci. Instrum. 75, 2229 (2004).

${ }^{8}$ I. Dufour, S. M. Heinrich, and F. Josse, J. Microelectromech. Syst. 16, 44 (2007).

${ }^{9}$ Z. Hu, T. Thundat, and R. J. Warmack, J. Appl. Phys. 90, 427 (2001)

${ }^{10}$ D. W. Dareing and T. Thundat, J. Appl. Phys. 97, 043526 (2005).

${ }^{11}$ S. Timoshenko, J. Opt. Soc. Am. 11, 233 (1925).

${ }^{12}$ M. J. Wenzel, F. Josse, S. M. Heinrich, E. Yaz, and P. G. Datskos, J. Appl. Phys. 103, 064913 (2008).

${ }^{13}$ G. G. Stoney, Proc. R. Soc. London, Ser. A 82, 172 (1909).

${ }^{14}$ R. W. Hoffman, in Physics of Thin Films, edited by G. Hass and R. E. Thun (Academic, New York, 1966), Vol. 3, p. 211.

${ }^{15}$ M. Calleja, J. Tamayo, A. Johansson, P. Rasmussen, L. M. Lechuga, and A. Boisen, Sens. Lett. 1, 20 (2003).

${ }^{16}$ M. Nordström, S. Keller, M. Lillemose, A. Johansson, S. Dohn, D. Haefliger, G. Blagoi, M. Havsteen-Jakobsen, and A. Boisen, Sensors 8, 1595 (2008).

${ }^{17}$ W. Flügge, Viscoelasticity, 2nd ed. (Springer-Verlag, New York, 1975),

${ }^{18}$ F. P. Beer, E. R. Johnston, and J. T. DeWolf, Mechanics of Materials, 4th ed. (McGraw-Hill, Boston, 2006).

${ }^{19}$ E. M. McCash, Surface Chemistry (Oxford University Press, Oxford, 2001).

${ }^{20}$ W. Haiss, Rep. Prog. Phys. 64, 591 (2001).

${ }^{21}$ N. V. Lavrik, C. A. Tipple, M. J. Sepaniak, and P. G. Datskos, Biomed. Microdevices 3, 35 (2001).

${ }^{22}$ S. Hyun, T. K. Hooghan, W. L. Brown, and R. P. Vinci, Appl. Phys. Lett. 87, 061902 (2005).

${ }^{23}$ T. Namazu, S. Inoue, K. Takio, T. Fujita, K. Maenaka, and K. Koterazawa, Proc.-18th IEEE International Conference on MEMS (2005), p. 447.

${ }^{24}$ R. Feng and R. J. Farris, J. Mater. Sci. 37, 4793 (2002). 
${ }^{25}$ O. J. Amu, S. Schneider, F. Josse, J. Hossenlopp, and Y. Jones, Proc.-IEEE Ultrason. Symp. 1649 (2005).

${ }^{26}$ B. Morray, S. Li, J. Hossenlopp, R. Cernosek, and F. Josse, Proc.-2002 IEEE International Frequency Control Symposium (2002), p. 294.
${ }^{27}$ J. D. Ferry, Viscoelastic Properties of Polymers, 3rd ed. (Wiley, New York, 1980) p. 608.

${ }^{28}$ N. G. McCrum, B. E. Read, and G. William, Anelastic and Dielectric Effects in Polymeric Solids (Wiley, New York, 1967), p. 105. 